Rabaska

Revue d'ethnologie de l'Amérique française

\title{
Suivre les traces pour trouver sa propre démarche. Réflexion autour du terrain ethnologique
}

\section{Pascal Huot}

Volume 13, 2015

Présence de Marius Barbeau : l'invention du terrain en Amérique

française. Autour d'un legs centenaire (1914-2014)

URI : https://id.erudit.org/iderudit/1033772ar

DOI : https://doi.org/10.7202/1033772ar

Aller au sommaire du numéro

Éditeur(s)

Société québécoise d'ethnologie

ISSN

1703-7433 (imprimé)

1916-7350 (numérique)

Découvrir la revue

Citer cet article

Huot, P. (2015). Suivre les traces pour trouver sa propre démarche. Réflexion autour du terrain ethnologique. Rabaska, 13, 315-324.

https://doi.org/10.7202/1033772ar d'utilisation que vous pouvez consulter en ligne. 


\section{Suivre les traces pour trouver sa propre démarche. Réflexion autour du terrain ethnologique}

Pascal HuOT

Ethnologue et photojournaliste indépendant

Marius Barbeau s'est immergé dans ses terrains ethnologiques, de ses jeunes années jusqu'à ce que le passage des ans ralentisse ses jambes alertes. De nos jours, il peut sembler plus difficile d'organiser un terrain comme le faisait cet illustre anthropologue alors que, frais sortis de l'université, nous attendent les contraintes financières et les engagements de toute sorte. Mais qu'en est-il donc de cette conception du terrain et de la relève ? Qu'en pensent les chercheurs d'aujourd'hui? ». À la demande des organisateurs du colloque, j'ai été invité à présenter mon approche du terrain ethnologique et ma méthode de travail.

Cette présentation se veut de ce fait foncièrement personnelle, teintée de mon expérience, de mes choix et de mes engagements. Elle porte donc sur le terrain, certes, mais un terrain qui sort du cadre universitaire ; un terrain parmi d'autres, appelé à évoluer selon les aventures. Plus prosaïquement, en tant qu'ethnologue indépendant, mon propos pourrait prendre l'allure d'une trousse à outils pour partir sur le terrain, ce qu'elle n'est évidemment pas !

Par ce regard porté sur ma méthode de travail, il sera possible de faire certains rapprochements avec celui de Marius Barbeau, célébré pour le centenaire de l'invention du terrain en Amérique française (1914-2014).

Le terrain ethnologique sera ainsi abordé dans un premier temps avec l'objectif de faire ressortir les bénéfices que cette expérience engendre pour ensuite se pencher sur la pratique d'un chercheur indépendant. Allant du choix du terrain à la diffusion des résultats, l'inévitable question monétaire sera également décortiquée, puisque pour vivre il faut vendre! La conclusion met en relief les propos par la démonstration d'un exemple concret avant de s'ouvrir vers une mise en relation avec le milieu journalistique. 


\section{Les bénéfices liés au terrain}

Avant de pouvoir retirer des bénéfices du terrain ethnologique, il importe d'avoir le goût du terrain, d'en assumer également les impératifs et conséquences. Il faut être en mesure de s'investir pleinement, accepter que l'on soit loin de sa famille et de ses amis durant certaines périodes ${ }^{1}$. Pourtant, le terrain se révèle souvent une aventure sur plusieurs plans : les découvertes, les relations humaines, l'expérimentation... Les savoirs et les savoir-faire ethnologiques ne peuvent pas toujours s'apprécier dans la distance. C'est pourquoi l'un des privilèges les plus heureux de l'ethnologue-baroudeur, de celui qui fait fi des aléas géographiques et logistiques de la vie de terrain, c'est de fouler le sol de l'Autre. Comme Pierre Perrault l'a si bien dit: « entre des souliers vernis puis une paire de bottes, j'ai toujours choisi la paire de bottes $^{2} »$. J'ai choisi mon camp, mon costume, en ayant goûté l'aventure.

L'avantage indéniable de passer du temps sur le terrain, outre celui de pouvoir y salir ses bottes, est l'immersion dans un milieu. Ce contact privilégié permet de recueillir divers types de sources, de l'enquête orale en passant par l'observation in situ. "Faire de l'enquête, c'est accumuler des informations de qualité sur des sujets, des personnes, des pratiques, des événements, en somme c'est construire son information en fonction d'une pertinence et d'une utilité ${ }^{3} \gg$. L'ethnologue aura donc le loisir d'expérimenter par tous ses sens, d'entrer en relation, de vivre un rythme parfois différent, de cerner d'autres réalités qui auraient pu lui échapper. Mais ces gains, pour les atteindre, commandent toutefois une condition : l'ouverture d'esprit et la disponibilité. Aussi, les bénéfices d'un terrain s'accompagnent par nécessité du temps, denrée tellement plus rare aujourd'hui lorsqu'elle se calcule en coût de projet et en jour-personne !

Aller à la source permet une rencontre directe avec les porteurs de tradition, de savoir et de savoir-faire. De ce vis-à-vis, des fils se créent. Ténus au début, le terrain doit permettre de les solidifier, de créer de la confiance. À terme, le développement d'un réseau de contacts, indispensable dans la poursuite du métier, contribue à mesurer les bénéfices du terrain.

En 2006, j'ai eu le privilège de participer au projet d'Inventaire des ressources ethnologiques du patrimoine immatériel (IREPI) ${ }^{4}$ au Bas-Saint-

1. Une problématique majeure lorsque l'on est jeune chercheur, c'est que les premières années qui suivent l'école coïncident souvent également avec le moment de la naissance de nos enfants. Le sacrifice en est d'autant plus grand, ce qui peut expliquer aussi pourquoi certains ethnologues, outre la sécurité d'emploi, souhaitent trouver un poste de bureau avec pied-à-terre de type ministériel ou muséal, contrairement aux ethnologues qui se spécialisent uniquement dans la collecte sur le terrain.

2. Michel La Veaux, Pierre Perrault parle de l'Île-aux-Coudres, Office national du film du Canada, 1999, $40 \mathrm{~min}$.

3. Martine Roberge, Enquête orale. Trousse du chercheur, Québec, Laboratoire d'ethnologie urbaine, CÉLAT, Faculté des Lettres, Université Laval, 1995, p. 7.

4. «La recherche a pour but de recenser les ressources ethnologiques du patrimoine immatériel 
Laurent et en 2008 au Saguenay-Lac-Saint-Jean. C'est véritablement par ces expériences que j'ai pris goût à l'immersion complète sur un terrain pour en faire un travail de longue haleine. Dès lors, à la suite de l'obtention de ma maîtrise en ethnologie des francophones en Amérique du Nord, j'ai commencé à monter un plan d'action pour pouvoir retourner sur le terrain par mes propres moyens. C'est ici que ma démarche se singularise un peu.

\section{La pratique d'un jeune chercheur indépendant Le choix du terrain}

Le choix du terrain d'enquête ne doit pas se faire dans un aléatoire imprécis, ou en utilisant une carte épinglée sur le mur de son bureau, laissant le hasard du dard décider du lieu d'investigation. Tout au contraire, comme au moment de lancer son entreprise, il est opportun d'élaborer un plan d'affaires ${ }^{5}$. En combinant l'objectif de la recherche ainsi que le choix du terrain, la structure de son entreprise se dessine. Pour en assurer la réussite, quelques éléments clés peuvent jouer un rôle déterminant.

À ne pas négliger, nos contacts préalables peuvent nous faire sauver énormément de temps et de ressources, surtout pour commencer! L'avenue la plus sécuritaire pour partir de manière autonome et à ses frais sur un premier terrain est sans doute de privilégier un lieu où l'on connaît des personnesressources qui pourront rapidement nous mener vers les informateurs recherchés ${ }^{6}$. Certains informateurs deviennent même nos ambassadeurs sur le terrain, ayant déjà quelquefois vendu notre salade avant même notre arrivée, ce qui facilite et augmente l'efficacité du temps passé sur le terrain. De mon côté, comme j'avais développé certains contacts au Saguenay-Lac-Saint-Jean avec le projet IREPI, je me suis dit que le Saguenay, déjà géographiquement assez important, serait mon premier terrain de recherche?

(IREPI) dans les régions du Québec. Le projet vise plus précisément à identifier et à reconnaître les porteurs de traditions ainsi qu'à valoriser leurs savoirs, savoir-dire et savoir-faire. Dans la foulée de la Convention pour la sauvegarde du patrimoine culturel immatériel adoptée par l'UNESCO en 2003, le projet IREPI, lui-même amorcé en 2004, contribue au renforcement du sentiment d'appartenance régionale tout en faisant connaître la diversité de la créativité humaine à l'ensemble du Québec ». Pascal Huot et Mathieu Tremblay, « Pour un patrimoine autre », La Quête, n 103, juin 2008, p. 26.

5. Cette planification inclut les coûts reliés à notre terrain : hébergement, nourriture, transport, équipement...

6. Différentes méthodes de recherche de contacts s'appliquent. Il est possible de communiquer avant le départ avec nos personnes-ressources, mais aussi avec les diverses associations d'histoire, cercles de fermières, curés de paroisse, pour faire un premier topo avec eux de nos objectifs et planifier des rencontres dès notre installation sur le terrain. Ce travail préalable peut être fait à distance. Ces prérencontres, souvent téléphoniques ou par courriel, nous permettent d'être déjà attendus sur le terrain. Les interlocuteurs peuvent déjà commencer à réfléchir à notre projet de leur côté, ce qui engendre régulièrement un effet cascade, car ils auront déjà en tête des noms de personnes qu'ils voudront nous présenter.

7. Quand c'est possible, certains pourraient être tentés de retourner dans leur région natale et utiliser le cercle familial pour trouver des informateurs. Mais, de mon côté, j'aime profiter de ce métier pour découvrir et en apprendre davantage sur des territoires nouveaux et inconnus. Être en immersion 
Il faut également s'assurer de la richesse du milieu. Celui-ci doit posséder un terreau fertile. Il faut savoir ce que l'on recherche et avoir une idée de qui peut combler nos attentes. Bien connaitre son terrain est primordial : être au fait de son histoire, de sa littérature et des principaux chercheurs qui $\mathrm{y}$ travaillent et sur quelle matière. Un terreau fertile ne s'épuisera pas : au contraire, il se bonifiera. À l'image de Marius Barbeau qui retournait souvent sur ses mêmes terrains de collectes, un seul passage ne peut suffire.

Dans le choix des sujets d'enquête, il faut continuellement garder en tête la diffusion des résultats. Ici, on n'est pas dans le dépôt d'un rapport pour une chaire de recherche qui commandite la collecte. L'ethnologue est seul avec sa matière brute, et il doit faire connaître et rentabiliser ses recherches ${ }^{8}$.

\section{L'autofinancement et la diffusion des résultats}

En tant que chercheur indépendant, il est impératif de pouvoir tirer un revenu de son terrain. De mon côté, j'ai décidé de partir, sans subvention, sans contrat de recherche commandité et sans l'aide logistique d'une université. Une complète liberté d'agir en toute indépendance qui vient cependant avec son lot de responsabilités. Il devient dès lors impératif que le terrain soit rentabilisable monétairement. L'idée est au rendement !

Pour ce faire, les informateurs forment une corne d'abondance en ce qu'ils détiennent moult sujets de connaissance. Lorsque l'on rencontre un Louis l'Aveugle ${ }^{9}$, on se presse d'agir comme Marius Barbeau. Celui-ci a exploité à son maximum l'information que son interlocuteur lui offrait. La richesse des savoirs et des savoir-faire transmis par les personnes rencontrées est souvent plus grande qu'elle n'y paraît à prime abord. Par la discussion, en passant du temps avec son informateur, il est possible de voir et de connaître son environnement, ses champs d'expertise et ses intérêts. Ce faisant, en plus de collecter sur un sujet donné, pourquoi ne pas l'interroger autrement? S'intéresser à ces personnes, partager avec elles nos intérêts, nos champs de recherche, être à l'écoute, voilà autant de façon d'établir la confiance. Cette confiance est primordiale et mène parfois ces mêmes personnes à nous référer à leur famille et à leurs amis pour collecter d'autres sujets, étant déjà dans le milieu.

De la parole aux actes, voici appliquée sur le terrain cette utilisation des informateurs avec l'exemple de Sainte-Rose-du-Nord ${ }^{10}$. Tout d'abord, j'ai

complète, hors du cercle familial, permet de se consacrer pleinement et entièrement à la recherche. C'est l'occasion de devenir un autre Marius Barbeau : « Il n'est pas question de parler de la pluie et du beau temps avec Marius Barbeau. Il s'intéresse à son travail, presque seulement à son travail ». Serge Gauthier, Marius Barbeau. Le grand sourcier, Montréal xyz éditeur, 2001, p. 35.

8. À ce titre, certains sujets se vendent plus difficilement. Par exemple, la chasse et la pêche constituent des sujets qui intéresseront généralement les revues et journaux à une certaine période de l'année, si elles ne sont pas spécifiquement dédiées à ces sujets.

9. Louis Simard dit l'Aveugle (1850-1918) : www.encyclobec.ca/main.php?docid=166.

10. La municipalité de Sainte-Rose-du-Nord est située dans le comté du Fjord-du-Saguenay. Lieu 
interrogé une personne clé, soit une dame âgée, qui a toujours vécu dans le petit village et qui s'investit dans la vie de celui-ci. À ce discours, j'ai jumelé celui plus officiel du maire du village. Plusieurs sujets ont ainsi été abordés. Il en est issu des articles très diversifiés ayant pour thème l'église paroissiale, un témoignage de l'incendie de celle-ci, l'histoire de la création du mobilier en bois, une initiative du curé, la place du tourisme et sa perception de la région ainsi que la petite chapelle de Tableau. Ces informateurs m'ont introduit à de nombreux sujets qui ont mené à la rédaction de plusieurs publications.

Si les informateurs constituent une richesse en connaissance, pour atteindre la rentabilité, il est nécessaire de transformer cette richesse première en salaire par la publication dans des journaux et magazines. Déjà pendant mes études de baccalauréat, j'avais cette volonté de diffusion de mes recherches. Je choisissais avec discernement pour mes travaux des sujets de recherche que je pourrais récupérer par la publication d'un article. De plus, à la maîtrise, avec l'aval de ma codirectrice, j'ai suivi un cours optionnel faisant la corrélation entre une expérience de terrain et la rédaction d'article scientifique. Cela a donné lieu à la publication de « Tourisme culturel en milieu insulaire, le cas de l'Île aux Grues " publié dans la revue Ethnologies ${ }^{11}$.

Les liens étaient donc établis avec le milieu de la rédaction et j'avais développé plusieurs contacts. Une sécurité qui a certes eu son importance dans mon plan d'affaires concernant la faisabilité d'un tel projet lorsqu'est venu le temps de me lancer à corps perdu sur mon premier terrain. J'avais pu ainsi m'assurer au préalable de la vente d'articles auprès de certains rédacteurs en chef.

Cependant, pour réussir à bien tirer son épingle du jeu et voir les bénéfices, entendons ici financiers, un autre atout consiste à trouver un créneau rentable, une niche inexploitée ou mal exploitée. Il faut penser hors des sentiers battus ${ }^{12}$.

De mon côté, cette niche, je l'ai trouvée avec le patrimoine religieux populaire. J'ai développé un créneau rentable avec la publication d'articles ethnologiques sur ce patrimoine dans des revues spécialisées. J'y devenais un contributeur intéressant par mon statut d'ethnologue, abordant des sujets religieux, mais avec un regard extérieur. Les rédacteurs en chef aiment le style plus humain et le choix des sujets différents.

\section{Les milieux de l'édition}

Dans le milieu de l'édition des magazines et des journaux, la méthode tra-

touristique reconnu, il se trouve sur la rive nord du fjord du Saguenay, entre Chicoutimi et Tadoussac.

11. Pascal Huot, « Tourisme culturel en milieu insulaire. Le cas de l'Île aux Grues » Ethnologies, vol. $31, n^{\circ} 1,2009$, p. 127-162.

12. Le photographe d'aventure Patrice Halley y va de ce conseil dans un article publié en 2009 destiné aux futurs photographes voulant publier dans des magazines. Patrice Halley, «Alors, vous voulez devenir photographe d'aventure ? $1^{\text {re }}$ partie », Photo Solution, vol. 2, no 5 , septembre 2009, p. $22-25$. 
ditionnelle pour vendre un article est d'écrire au rédacteur en chef en lui soumettant quelques synopsis. Par la suite, selon le choix de celui-ci, le journaliste travaille le sujet. Cette méthode est souvent incompatible avec la logistique du terrain. Pour cette raison, j'ai plutôt opté pour la réalisation de la recherche de terrain, l'écriture des articles en fonction du style de la revue ciblée et l'envoi de l'ensemble en soumission après entente avec le rédacteur en chef.

Par contre, il faut savoir à quel public on destine ses textes et quel est le type de publication visée. Chaque type entraîne un exercice d'écriture différent. «Toutefois, pour réussir, il faut savoir à qui l'on s'adresse et bien connaître tous les arcanes de la presse québécoise ${ }^{13}$ ». La photographie a également un impact non négligeable. Les rédacteurs en chef aiment bien les articles clés en main avec de bonnes photos. L'image est aussi importante que le texte. Trouver des images pour illustrer un article est souvent une tâche ardue pour ces revues qui n'ont généralement pas de directeur photo. De plus, un magazine ne souhaitera pas nécessairement engendrer des frais supplémentaires pour aller photographier notre informateur, la pratique, le lieu, etc. Notre méthodologie d'ethnologue de terrain devient ici un atout très intéressant : nous photographions nos sujets dans l'action ou dans leur milieu. Nos photographies, en plus de constituer des sources premières, témoignent de notre enquête et ont ainsi une valeur ajoutée à notre soumission d'article.

Afin d'illustrer les possibilités de publications et les distinctions entre ces écrits, j'ai procédé à une subdivision en trois grands groupes : les articles grand public (généralistes ou spécialisés), les petits journaux locaux et les articles scientifiques.

Les articles grand public regroupent les textes publiés dans divers journaux et revues destinés à un lectorat général dont le contenu est d'intérêt collectif, d'information ou de vulgarisation. Règle générale, ces publications constituent celles où les revenus sont les plus élevés. Je tire l'ensemble de mes revenus de terrain de ce type d'article. Ces publications paient leurs contributeurs à titre de journaliste pigiste ${ }^{14}$. Il s'agit d'un milieu où règne une bonne compétition, mais où il demeure possible de faire sa place. Et comme le souligne si bien le journaliste indépendant Ludovic Hirtzmann :

Dans le monde de la pige, un mythe a la vie dure : « Il n'y a pas de travail pour les pigistes. »C 'est faux. Certes, le bassin médiatique est plus petit, en nombre, au Québec, qu'aux États-Unis ou en France, mais toutes proportions gardées, les possibilités ne manquent pas. Le nombre de pigistes est même proportion-

13. Ludovic Hirtzmann, Vivre la pige! Guide pour les journalistes indépendants, Québec, Éditions MultiMondes, 2004, p. XI.

14. Je n'entrerai pas ici dans une énumération des tarifs, variant d'un endroit à un autre, payable au feuillet ou aux mots. À ce titre, l’Association des journalistes indépendants du Québec (Aлю) publie une liste non exhaustive des tarifs à la pige des médias québécois : www.ajiq.qc.ca/Tarifs2010v4.pdf. 
nellement moins important qu'en France. Les rédacteurs en chef se plaignent depuis toujours de manquer de bons pigistes. Donnez-leur tort ! ${ }^{15}$

Pour ce qui est des petits journaux locaux ${ }^{16}$, ceux-ci paient rarement (jamais !) leurs auteurs ; par contre, j’y publie néanmoins mes résultats de collectes. J'applique ainsi la notion de recherche-action en redonnant à la communauté les fruits de mes recherches et en les rendant accessibles à la population locale. Et il ne faut pas s'y méprendre, ce sont souvent les articles avec lesquels on retire le plus de retombées. Ces articles parus dans ces petites publications sont lus par les informateurs et généralement par leur entourage, ce qui engendre une sorte de reconnaissance et de valorisation pour ceux-ci. L'autre avantage, lorsque l'on est sur le terrain, est que si un informateur a vu passer des articles précédemment, il est plus enclin à ouvrir sa porte. En somme, cela permet de se faire connaître par les gens du milieu : une forme de publicité détournée !

Enfin, je publie également des articles scientifiques, me permettant ainsi de rassembler l'ensemble de l'information recueillie sur le terrain et d'y réfléchir selon un objectif déterminé. À l'inverse des articles grand public, souvent courts, on peut ici donner plus de détails, aller plus profondément dans l'analyse. C'est l'occasion de faire un travail d'ethnologue universitaire à proprement parler et d'obtenir également une reconnaissance de mes pairs sur mes travaux.

On a reproché à Marius Barbeau d'écrire des articles grand public et non exclusivement des articles scientifiques. Cela ne l'a jamais arrêté, bien au contraire. Une telle pratique ne discrédite en rien ses recherches. L'ethnologue Serge Gauthier résume bien cette idée et l'importance que Marius Barbeau accorde à ses publications dites grand public :

Il faut que tu publies des articles dans de pauvres journaux de fin de semaine afin de nous aider à remplir nos obligations, pauvre Marius. Tu travailles comme simple pigiste. Ce n'est pas ça qui va faire grandir ta réputation de chercheur. Et c'est sans intérêt, toutes ces anecdotes ! Marius Barbeau n'est pas d'accord. Il aime bien diffuser ses recherches dans Le Petit journal, dans La Patrie, dans La Revue moderne ${ }^{17}$.

J'ai adopté cette vision, en faisant exactement la même chose : sur un sujet donné, je publie des articles grand public comme pigiste pour avoir de l'argent en retour et des articles scientifiques pour faire grandir ma réputation de chercheur!

15. Ludovic Hirtzmann, op. cit., p. XI.

16. Ces journaux sont souvent produits par le village, la MRC ou les associations locales. Y sont exclus les hebdos régionaux qui paient leurs collaborateurs et constituent une source de revenus.

17. Serge Gauthier, op. cit., p. 63-64. 
Opportunisme, diront certains ! Et pourtant, il faut développer une méthode efficace pour parvenir à des publications multiples. La méthodologie du recyclage, une approche développée notamment par le journalisme indépendant Jean-Benoît Nadeau dans ses conférences et dans son livre Écrire pour vivre: conseils pratiques à ceux qui rêvent de vivre pour écrire ${ }^{18}$, repose sur le potentiel infini de la récupération. En résumé, il propose de vendre le même article à plusieurs magazines et journaux en changeant l'entête et en réaménageant le texte en fonction de la publication visée. C'est une manière de rentabiliser facilement la recherche et d'augmenter les revenus de terrain de manière exponentielle.

À titre d'exemple, cette approche mercantile et scientifique a été utilisée en 2009 pour la question des Métis au Saguenay-Lac-Saint-Jean. En plus de publier des articles grand public et généralistes sur la question métisse au Québec, j'ai publié un article scientifique dans la revue Rabaska ${ }^{19}$, repris ensuite dans la revue Saguenayensia ${ }^{20}$, lequel permettait de faire le point sur l'avancée de mes recherches sur le sujet en plus d'offrir un bilan ethnohistorique sur la communauté des Métis de la Boréalie.

\section{Conclusion}

Le terrain ethnologique, c'est par essence même une aventure. Lorsqu'on s'y laisse prendre, le dénouement est toujours incertain. « Aujourd'hui comme hier, le terrain est également le lieu où les réalités du présent côtoient les traces du passé et les signes du futur. En ce sens, il se donne comme un riche réservoir de ressources pour les hommes et les femmes de science qui étudient les productions de leurs semblables à travers le temps ${ }^{21} »$.

L'expérience du terrain en tant qu'ethnologue indépendant n'est certes pas pour tout le monde, je le concède. On doit avoir les reins solides et avoir une confiance immense en son potentiel. Le jeu est risqué, voir téméraire pour certains, mais pas plus qu'un entrepreneur qui se lance en affaires. Il faut voir sa personne comme une petite entreprise. La liberté a son prix, il faut être capable de vivre dans l'incertitude monétaire et, ici c'est vraiment le cas de le dire, c'est sur le terrain que l'on apprend réellement ${ }^{22}$.

18. Jean-Benoît Nadeau, Écrire pour vivre : conseils pratiques à ceux qui rêvent de vivre pour écrire, Montréal, Québec Amérique, 2007, 416 p.

19. Pascal Huot, «Les Métis de la Boréalie. Une présence autochtone au Québec », Rabaska, vol. 8, 2010, p. 77-92.

20. L'article publié précédemment dans la revue Rabaska a été repris à la demande du rédacteur en chef invité qui préparait la sortie d'un numéro consacré à l'éveil de la voie autochtone en Boréalie. Pascal Huot, «Les Métis de la Boréalie. Une présence autochtone au Québec », Saguenayensia, vol. 52, $\mathrm{n}^{\mathrm{o}}$ 1-2, janvier-juin 2011, p. 29-36.

21. Jean Simard, Le Québec pour terrain. Itinéraire d'un missionnaire du patrimoine religieux, Québec, Les Presses de 1’Université Laval, 2004, p. 1.

22. Pour ceux qui souhaiteraient se lancer dans une telle aventure, outre le fait de vous y encou- 
Pour ma part, le goût du terrain est fusionné au goût de l'écriture et à la passion de la photographie. Pour trouver mon financement, la publication est devenue une alliée de choix. Mes pas m'ont mené vers des rencontres fructueuses ; mes écrits ont consolidé mes informations, chacun selon ses particularités.

Voici un exemple éloquent : le calvaire de Shipshaw ${ }^{23}$. C'est d'abord par un réseau de contacts que j'ai été mis au courant de cette histoire. Une amie historienne de la région du Saguenay m'a mis en relation avec une dame qui pourrait sans doute m'en apprendre un peu plus sur cette croix de chemin, car elle était amie de la famille qui l'a érigée. À la suite d'une première rencontre, celle-ci m'a présenté à l'instigatrice du déplacement et de la revitalisation de la croix. Celle-ci deviendra ma principale informatrice sur le sujet. La collecte devenait des plus urgentes, car cette dame était âgée et malade. Plusieurs versions du sujet ont été publiées dans des revues grand public dites religieuses, notamment dans Notre-Dame du Cap ${ }^{24}$. Ensuite, l'enquête a donné lieu à une publication scientifique dans la revue Rabask ${ }^{25}$. Et à titre de recherche-action, comme l'informatrice était lectrice de la revue d'histoire locale et déplorait que jamais celle-ci n'ait traité de la croix en question, l'article a été repris dans la revue Saguenayensia ${ }^{26}$.

L'ethnologue, par sa formation, a des atouts majeurs pour se tailler une place dans différentes sphères d'activité professionnelle. Le monde du journalisme est l'un de ceux-ci. Ce milieu est en pleine mutation depuis quelques années : les salles de rédaction diminuent leur personnel, le télétravail fait maintenant partie intégrante du métier, on fait de plus en plus appel à des journalistes pigistes, les journaux se livrent une bataille constante pour avoir du matériel inédit, la nouvelle est vite saturée par la toile, les rédacteurs en chef de magazine souhaitent et doivent offrir autre chose au lectorat. « Dans le monde du journalisme, cela se traduit souvent par un recours aux journalistes

rager pleinement, vous trouverez sur les sites internet des deux principaux regroupements de journalistes du Québec, une mine d'information, soit : la Fédération professionnelle des journalistes du Québec (FrıQ), www.fpjq.org; et l'Association des journalistes indépendants du Québec (AısQ), www.ajiq.qc.ca.

23. Résumé tiré de Rabaska, revue d'ethnologie de l'Amérique française, vol. 10, 2012, p. 349 : «Témoins d'une ferveur religieuse populaire, les croix de chemin et calvaires signent le paysage du Québec. Vestiges d'une époque, ils ont subi pour plusieurs les contrecoups du temps. Mais certains jouissent encore aujourd'hui de la dévotion de fidèles. À cet égard, le calvaire de Shipshaw dans la ville de Saguenay, vieux de plus de 61 ans, mérite considération. D'abord et avant tout, témoin d'une histoire humaine, sa survie et sa revitalisation sont tributaires des efforts d'une dame, Charlotte MercierBouchard. [L'enquête ethnologique] retrace la chronologie de ce calvaire, son édification, ses épreuves ainsi que les actions posées pour en assurer la revitalisation et la pérennité. »

24. Pascal Huot, "Le Calvaire de Shipshaw, une histoire à raconter ", Notre-Dame du Cap, vol. 121, juin 2012, p. 8-9.

25. Pascal Huot, "Grandeurs et misères du calvaire de Shipshaw », Rabaska, vol. 10, 2012, p. 81-95.

26. Pascal Huot, « Grandeurs et misères du calvaire de Shipshaw », Saguenayensia, vol. 54, n ${ }^{\circ}$, 2013, p. 18-25. 
indépendants, qui représentent une solution simple, efficace, pratique, et très économique de présenter un contenu inédit, de qualité et innovateur ${ }^{27} \gg$. C'est ici qu'entre en ligne de compte tout le potentiel d'une approche ethnologique du sujet. L'ethnologue explore généralement des sujets plus intemporels, ce qui permet aux rédacteurs de se servir facilement des textes en tout temps.

L'approche de l'ethnologue lui procure un avantage dans le milieu de l'écrit. Il ne porte pas le même regard qu'un journaliste sur un sujet donné, ayant tendance à questionner au-delà des questions primaires (qui ? quoi ? quand ? où ? comment ? pourquoi ? ${ }^{28}$. Plus qu'une simple citation, l'informateur prend véritablement part au propos, il transcende l'article.

L'ethnologue a sa force également en photographie ${ }^{29}$. En photojournalisme, il est nécessaire d'entrer en relation rapidement avec la personne. Il faut réussir à obtenir sa confiance si l'on souhaite faire une bonne photo. On doit aborder les gens de manière « plus humaine », et non dans une action de vol d'image, qualité inhérente au métier d'ethnologue. De plus, en ethnologie, on ne photographie pas l'informateur sans le mettre en contexte ou en action. Cette façon de faire permet de contextualiser les images, offrant mieux au rédacteur en chef que la photo passeport de l'informateur. Il est ainsi possible de transmettre une compréhension globale d'une situation, qualité indispensable en photojournalisme.

Bref, l'ethnologie constitue une discipline qui ouvre de multiples avenues. Elle outille ses étudiants pour leur permettre de développer leurs ressources et leur intelligence. Mais surtout, « la formation ne remplace pas l'expérience ${ }^{30} »$. Si je devais donner un conseil au futur ethnologue-étudiant, allez sur le terrain, même pour de courtes périodes, sortez, faites des entrevues, apprenez, mais aussi diffusez vos résultats, écrivez des articles, participez au journal de votre université, aux journaux locaux, allez parler de vos recherches à votre radio étudiante. Il est possible de tirer un article de chaque entrevue que vous collectez. Aiguisez votre plume. À la sortie de l'université, vous ne serez pas démuni, vous aurez déjà commencé à faire votre nom dans la discipline, vous aurez déjà une connaissance du milieu de l'édition. Vous ferez face à toute éventualité, mais serez fin prêt à l'affronter sur le terrain.

27. Pascal Lapointe et Christiane Dupont, Les Nouveaux Journalistes. Le guide. Entre précarité et indépendance, Québec, Les Presses de l'Université Laval, 2006, p. XIV.

28. En ethnologie, l'enquête et la collecte peuvent prendre plusieurs heures, voire plusieurs jours ; la manne d'information collectée en est de fait plus imposante, une pratique qui, outre le journalisme d'enquête, est incompatible avec l'idée de rapidité que demande la nouvelle quotidienne.

29. Bien sûr, il faut connaître et maîtriser les appareils photo.

30. Pascal Lapointe et Christiane Dupont, op. cit., p. 256. 